\title{
Treatment Patterns, And Effectivness of Anti- Leishmaniasis Agents for Patients with Cutaneous Leishmaniasis at Boru Meda Hospital, South Wollo, North East Ethiopia, 2017/18
}

Tsehai Seife $^{1}$, Alie Ayal ${ }^{2}$, Mastewal Misganaw ${ }^{3}$ and Feleke Tilahun Zewdu ${ }^{4^{*}}$

${ }^{1}$ Dermatovenereologiest, St Paul Millennium teaching hospital, Addis Ababa, Ethiopia

${ }^{2}$ BSC, MPH, Public health, Amhara regional health Bureau PHEM officer, Bahir Dar, Ethiopia

${ }^{3}$ B.Ed, M.Ed in TEFL, Bahir dar University, Amhara, Ethiopia

${ }^{4} B s c$, Msc in tropical dermatology, Boru Meda Hospital, Dessie, Ethiopia

Received: May 31, 2018; Accepted: June 13, 2018; Published: June 25, 2018

*Corresponding author: Feleke Tilahun Zewdu, Bsc, Msc in tropical dermatology, Boru Meda Hospital, Dessie, Ethiopia, E-mail: momflk@gmail. com

\section{Abstract}

Background: Cutaneous leishmaniasis is one of the endemic and neglected diseases known to exist in Ethiopian highlands. However, a neglected tropical disease overshadowed by lack of effective anti-leishmaniasis agent in Ethiopia. Thus, high number of population is faced for various degree of socio-economical and psychosocial morbidity. Hence, this study was initiated and conducted from July-February, 2017/18 to assess the patterns and effectiveness of different types of anti-leishmaniasis agents in Boru Meda Hospital, Dessie District.

Methods: A cohort study design was employed in six treatment categories via randomly allocated cutaneous patients from three clinical types at Boru Meda hospital Dermatology department. Detailed clinical assessment, biopsy/fine needle aspiration cytology, and skin slit smear leishmania parasite detection were done to confirm clinical suspension. Then, the intended treatment types were administered for three cycles. Finally the data were analyzed using Epi- info, SPSS and the results presented using graphs and tables.

Results: Among patients with mucocutaneous leishmaniasis who has took Systemic Sodium Stibogluconate (SSG) with Intra-Lesional SSG (IL SSG) the cure rate was $85.7 \%$, systemic SSG with allopurinol was as effective as $78.6 \%$. Patients with diffused cutaneous leishmaniasis who took both systemic SSG with allopurinol had an 80\% cure rate and systemic SSG and local therapy both cryotherapy and IL SSG had a clinical cure rate of $85.7 \%$. In addition, patients who diagnosed as localized cutaneous leishmaniasis and took only cryotherapy had $92.3 \%$ cure rate where as those patients who had a combined local therapy of both cryotherapy and IL SSG therapy showed clinical cure rate of $96.1 \%$.

Conclusion: As our study showed for any clinical type of cutaneous leishmaniasis, administering combined forms (Pentavalent antimonial with local therapies i.e. cryotherapy or/and IL SSG) of anti-leishmaniasis agents had a better cure rate than single therapies.

Keywords: Cutaneous leishmaniasis; Pattern of treatments; Effectiveness; Ethiopia;

\section{Author Summary}

Most cases of cutaneous leishmaniasis in Ethiopia are caused by L. aethiopica. Among these nearly $100 \%$ are localized cutaneous leishmaniasis, more than $80 \%$ mucocutaneous leishmaniasis and more than $75 \%$ diffused cutaneous leishmaniasis treated with pentavalent antimonials (SSG) having a great cure rate when it was in a combined form with local therapies than a single pattern.

\section{Introduction}

Cutaneous Leishmaniasis (CL) is a chronic, neglected tropical infectious disease caused by a group of protozoan parasites of the Leishmania genus. The parasites are transmitted to humans via the bite of phlebotomine sand flies and predominantly target reticulo-endothelial cells [1-3].
Cutaneous leishmaniasis was first described in Ethiopia by an Italian epidemiologist Martogilo in 1913. CL is known by different vernacular name in different localities of Ethiopia such as: "Volbo" in Ocholo, "Finchoftu" in central Shoa, "Kunchir" in Gojam, Gonder and parts of Wollo, "Giziwa" in Tigray, "Chewie" in Sodo, "Simbirahalkani" in Wollega and "Shahegne" in north Shewa [3].

Cutaneous leishmaniasis can present with a spectrum of clinical manifestations. Ulcerative skin lesions occurring at the site of the bite of the sand fly is the most common cutaneous manifestation (localized CL-LCL). While usually healing spontaneously after several months, it remains disfiguring and stigmatizing and often heals with scarring. There are several more rare forms like diffuse CL (DCL), and muco-cutaneous leishmaniasis which is often difficult to treat $[1,4,5]$. 
Most cutaneous leishmaniasis lesions are self-limiting and may heal in 1 - 5 years. In spite of this, treatment is justified in a variety of cases, namely early lesions, multiple lesions, lesions involving cosmetically sensitive sites, mucosal lesions, disseminated lesions and patients with significant immunosuppression $[2,6]$.

The disease still presents a therapeutic problem in several parts of the world. To-date, there is no safe, simple, cheap and effective ambulatory treatment for cutaneous leishmaniasis except meltifosine though not available in Ethiopia. Pentavalent antimony compounds, "the best drug of a bad bunch" still remain the mainstay of treatment in the majority of cases. Antimony compounds have the disadvantage of both toxicity and clinical resistance in at least $40 \%$ of cases in certain regions where they have been in use for a long time $[6,7,8]$.

Cutaneous leishmaniasis in the Old World is predominantly caused by L.tropica and L major, it is still estimated that several ten thousands of cases are due to L. aethiopica. These predominantly occur in Ethiopia, and more exceptionally in Kenya. Within Ethiopia, the annual cutaneous leishmaniasis burden is estimated at around 20.000 to 40.000 cases per year, of which $99 \%$ is thought to be due to L. aethiopica $[9,10]$. A recent study estimated almost 30 million of Ethiopians to be at risk for CL. CL in Ethiopia is a zoonotic disease, mainly occurring in the highland regions, involving rocky environments [11].

The classic therapy for all forms of leishmaniasis uses pentavalent antimonials as Sodium Stibogluconate (SSG) and Meglumine Antimoniate (MA) administered intravenously or intramuscularly. Other systemic treatments used are Amphotericin B deoxycholate (AB) and Liposomal Amphotericin (LAB), both intravenously, and intramuscular paromomycin and pentamidin. Local treatments based on intralesional pentavalent antimonials, topical paromomycin, thermotherapy, or cryotherapy is used for certain cases of cutaneous leishmaniasis. But as few studies in the New World cutaneous leishmaniasis revealed that the combined therapies between systemic Sodium Stibogluconate (SSG) with allopurinol, ketoconazol, cryotherapy and intra-lesional SSG showed promising effects for patients with cutaneous leishmaniasis [12-14]. Thus, this study was aims to assess three patterns of treatment and its effectiveness among patients with six categories of cutaneous leishmaniasis for treatment type under study.

\section{Methods}

This research was conducted from July-February, 2017/18 in Boru Meda hospital, $10 \mathrm{~km}$ away from Dessie district located in eastern zone of Amhara National Regional State at the north eastern edge of the Ethiopian highlands $411 \mathrm{~km}$ from the region capital city and $470 \mathrm{~km}$ north of Addis Ababa (capital of the country) situated between 11007'21.33"N, 39038'05.87' $\mathrm{E}$ with an elevation of 2,706 meters $(8,878 \mathrm{ft})$ above sea level.

Boru Meda hospital was established by Sudan Interior Mission (SIM) in 1955. The hospital is landed in a field bounded by mountain especially in the west and north direction in addition to the mountain. The primary objective at time of establishment of the hospital was focusing to give care on ophthalmology and dermatology services. Thus, it serves on both service areas for more than 40 years but now the hospital gives a comprehensive service, i.e., emergency, outpatient service and gynecology and obstetrics and inpatient service with 140 beds among these 45 beds was assigned for dermatology ward. Regarding human resource, there are 9 specialists among these, 2 of them are dermatologists and an adequate number of all the other health professionals constitute the health care team.

The hospital provides serves for a total of 2.5 million catchment population of south Wollo, North Wollo, Oromia especial zone, South Tigray and Afar region. In addition to the dermatologic cases diagnosis and treatment the hospital is used as a training center for health professionals in the surrounding health facilities and used as internship and attachment site for Wollo university medical department student.

This research was conducted on the treatment patterns, outcomes and effectiveness for intervention of various forms of cutaneous leishmaniasis. A total of 97 cutaneous leishmaniasis (MCL 28, LCL 52 and DCL 17) patients in Boru Meda hospital, dermatology department in three outpatients departments between July-February, 2017/18 one who come for the seek of curative, preventive and rehabilitation services to the hospital.

During this eight months study period, 97 patients were included in the study. 82 patients enrolled in the study whose skin slit smear was positive, 9 patients had negative skin slit smear and suggestive Fine Needle Aspiration Cytology (FNAC) result and the rest 6 patients were included after clinically diagnosed. One patient was excluded from the study at the beginning of the study due to deviated renal and liver function test.

Though Sodium Stibogluconate is recommended for first cycle (28 days) up to five to seven repeated cycle of the treatment cycle specially patients with MCL and DCL. Thus, patients were categorized in six different treatment patterns. Then, these patients were categorized randomly based on their clinical category and drugs took as; LCL (26 only Cryotherapy, 26 combined intra-lesional SSG with Cryotherapy), MCL; (14 allopurinol with SSG intramuscular (IM) or intravenous (IV), 14 combined SSG IV/ IM with intralesional SSG) and DCL; (10 allopurinol with SSG IM or IV, 7 combined SSG IV/IM, Cryotherapy, and intralesional SSG). Then, the patients were assessed for the clinical improvement or cure after providing respective treatment options for three cycles (of SSG therapy) or 90 days while admitting them in Boru Meda Hospital.

Meantime, those patients who took systemic SSG were had regular base line (CBC, SGOT, SGOPT, ALP, BUN, Creatinine, electrolyte and ECG for children who are less than ten years) at the beginning and every a couple of weeks with daily vital sign and conducting a grand round every week with ward nurses, seniors and daily by assigned nurses who follow those cases on treatments which were available in the hospital.

Besides, all findings laboratory findings, deviated vital signs, clinical improvements, patients complain and drug took were 
recorded in the data sheets for respective clinical groups every week after grand round.

Finally, the clinical variables, drug took, socio-demographic data and outcomes were entered in to Epi-info and then for the seek of analysis it was export in to SPSS. Then the SPSS data will be analyzed to show the difference and similarity via ANOVA Then, the outcome variables were presented through tables and charts.

\section{Definition}

"Clinical cure" was defined as complete epithelialization or visually healed at $2 \pm 1$ month after completion of therapy.

"Clinical response"-The response of the leishmanial skin lesions was determined at the end of therapy and at the follow up every month, 2nd and 3rd month.

"Clinical improvement" was defined as 75\%-99\% reepithelialization (for non ulcerative lesions or/and 75\%-99\% decrease in the size of the initial lesion).

"Clinical failure" was characterized as less than complete epithelialization or visually not healed at $2 \pm 1$ month after treatment completion.
LCL (Localized Cutaneous Leishmaniasis): Nodular or indurated lesion which may have ulcerated at the center, with a raised and erythematous edge around it. There may be several of these lesions close to each other, and they may spread into each other to form one large lesion.

MCL (Mucocutaneous Leishmaniasis): Lesions marked by involvement of naso-oral and pharyngeal mucosa.

$\boldsymbol{D C L}$ (Diffuse CL): This is a chronic, progressive condition that starts with few papular or nodular lesions followed by a gradual dissemination of the infection leading to multiple papular, nodular and plaque lesions involving larger areas of the skin that often do not ulcerate. Lesions are polyparasitic and resemble lepromatous leprosy.

\section{Result}

\section{Socio-Demographic Data}

Most of the study subjects who were exposed for various forms of therapy were male, 62(63.9\%), age ranges from 16-45 years(SD 23 \pm 1.23$), 43(44.3 \%)$ and $71(73.2 \%)$ patients were from rural area ( $44.3 \%$ or $73.2 \%$ ) with high numbers of patients with cutaneous leishmaniasis coming from Dessie town, zonal city of South Wollo zone. Moreover, 23 (23.7\%) of patients under study was illiterate, and $51(52,3 \%)$ were farmer with income source of farming and pastoralist (See Table 1).

Table1: Characteristics of cutaneous leishmaniasis cases who received treatment in BoruMeda Hospital, Dessie, Northeast Ethiopia, 2017.

\begin{tabular}{|c|c|c|c|c|}
\hline \multirow{2}{*}{ Ser. No } & \multirow{2}{*}{ Variables } & \multirow{2}{*}{ Characteristic } & \multicolumn{2}{|c|}{ Frequency } \\
\hline & & & $\mathbf{N}$ & $\%$ \\
\hline \multirow{3}{*}{1} & \multirow{3}{*}{ Age } & $1-15$ & 33 & 35 \\
\hline & & $16-45$ & 45 & 45.4 \\
\hline & & Above 45 & 19 & 19.6 \\
\hline \multirow{4}{*}{2} & \multirow{4}{*}{ Educational Status } & Illiterate & 23 & 23.7 \\
\hline & & Grade1-8 & 41 & 42.3 \\
\hline & & Grade9-12 & 26 & 26.7 \\
\hline & & Diploma and above & 7 & 7.3 \\
\hline \multirow{2}{*}{3} & \multirow{2}{*}{ Address } & Rural & 71 & 73.2 \\
\hline & & Urban & 26 & 26.8 \\
\hline \multirow{3}{*}{4} & \multirow{3}{*}{ Family Income } & Merchants & 23 & 23.7 \\
\hline & & Civil servants & 14 & 14.4 \\
\hline & & Others & 9 & 9.6 \\
\hline
\end{tabular}

\section{Clinical Characteristics}

The patients were admitted to the hospital for a standardized period of time for up to three cycles of the standard therapy with a couple of weeks rest between the cycles. Thus, all patients were complete their respective treatment patterns except one patient whose renal and liver function tests elevated more than three times to the normal range was excluded to the study at the beginning of the study.
In addition, 33 (34\%) of the patients who were included to the study had history of herbal application with significant scar around the lesions (Leishmania recidivans). But the rest 64 (66\%) patients did not have history of neither any herb application nor any medicine from the health institution (See table 2) and (See table 3). 
Table 2: Diagnostic methods used forcutaneouse leishmaniasis cases detection who received treatment in BoruMeda Hospital, Dessie, Northeast Ethiopia, 2017/18.

\begin{tabular}{|c|c|c|c|}
\hline \multirow{2}{*}{ Ser. No } & \multirow{2}{*}{ Investigation type } & \multicolumn{2}{|c|}{ Result (N, \%) } \\
\hline & & Total tested & Positive \\
\hline 1 & Skin slit smear & $97(100 \%)$ & $82(85 \%)$ \\
\hline 2 & Biopsy/FNAC* & $15(15.5 \%)$ & $6(40 \%)$ \\
\hline 3 & Clinical diagnosed & 9 & \\
\hline 4 & Total & 97 & \\
\hline
\end{tabular}

A total of 97 cutaneous leishmaniasis with three clinical types (MCL 28, LCL 52 and DCL 17), and six therapeutic categories: LCL (26 only Cryotherapy, 27 combined intra-lesional SSG with Cryotherapy), MCL; (14 only SSG intramuscular (IM) or IV, 14 combined SSG IV/IM with intralesional SSG) and DCL;
Table 3: Clinical characteristics for cutaneous leishmaniasis cases who received treatment in BoruMeda Hospital, Dessie, Northeast Ethiopia, $2017 / 18$.

\begin{tabular}{|c|c|c|c|}
\hline \multirow{2}{*}{ Ser. No } & \multirow{2}{*}{ Clinical variables } & \multicolumn{2}{|c|}{ Frequency } \\
\cline { 3 - 4 } & & N & \% \\
\hline 1. & Scar lesions & 41 & 42.3 \\
\hline 2. & Herbal application & 33 & 34 \\
\hline 3. & Previous Rx Hx & 37 & 38 \\
\hline 4. & Co-morbid & 6 & 6.2 \\
\hline
\end{tabular}

(10 only SSG IM or IV, 7 combined SSG IV/IM, Cryotherapy, and intralesional SSG).

Among those cutaneous leishmaniasis patients who were admitted and took drugs in six different clinical types had an overall clinical cure rate of $94.8 \%$ with respective improvement (See figure 1).

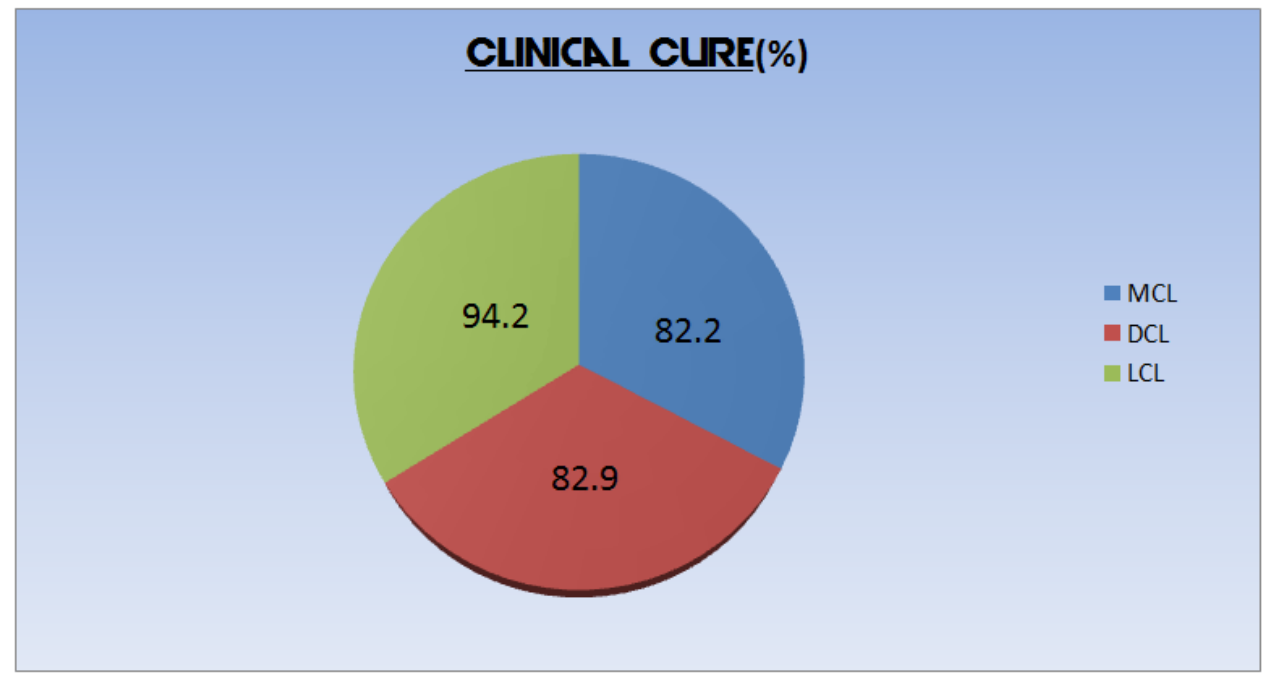

Figure 1: A pie chart shows the clinical cure rate of anti-leishmaniasis within the three cycles of treatment regimens in BoruMeda Hospital, Dessie, North east Ethiopia, 2017.

Those 14 patients with mucocutaneous leishmaniasis who have took systemic sodium stibogluconate with intralesional SSG $85.7 \%$ was improved, while as the clinical cure rate for those patients who took systemic SSG with allopurinol was 78.6\%.

For patients who diagnosed as diffused cutaneous leishmaniasis, 10 patients were admitted and took both systemic SSG and allopurinol and the curative rate was as high as $80 \%$ where as those 7 patients who took systemic SSG and local therapy with both cryotherapy and IL SSG had a clinical cure rate of $85.7 \%$ (See table 4 ).

A total of 52 patients who diagnosed as localized cutaneous leishmaniasis and took local therapy provide different clinical improvements in a combined and single treatment pattern. Twenty-six 26 patients who had only cryotherapy showed clinical cure of $92.3 \%$ at the $3 r d$ dose where as the rest the 26 patients had a combined cryotherapy and IL SSG therapy with a cure rate of $96.1 \%$.

\section{Discussion}

Though the magnitude of cutaneous leishmaniasis is not yet precisely known in Ethiopia, it results in numerous socio-economical and psychosocial effects on the population. Moreover, all the available anti-leishmania agents are considered as ineffective for leishmania aethiopica $(2,3)$. But cutaneous leishmaniasis patients who are admitted and as out or inpatients for various patterns of treatment showed promising cure rate where agents are administered as a single and combined ways.

Patients with Mucocutaneous Leishmaniasis (MCL) who have took systemic sodium stibogluconate via intra muscular or/ and intravenous with weekly intralesional pattern was as effective as 

$2017 / 18$

Table 4: Treatment patterns of cutaneous leishmaniasis cases in BoruMeda Hospital, Dessie, Northeast Ethiopia, 2017-2018.

\begin{tabular}{|c|c|c|c|c|c|c|c|c|c|c|c|}
\hline \multirow{3}{*}{ Ser. No } & \multirow{3}{*}{ CL Types } & \multirow{3}{*}{ Treatment Regimen } & \multicolumn{9}{|c|}{ Clinical response/Cure } \\
\hline & & & \multirow[t]{2}{*}{$\mathbf{N}$} & \multicolumn{2}{|c|}{$\begin{array}{c}\text { 1st } \\
\text { dose }\end{array}$} & \multicolumn{2}{|c|}{ 2nd doses } & \multicolumn{2}{|c|}{ 3rd doses } & \multirow{2}{*}{$\begin{array}{c}\text { Cure rate } \\
\%\end{array}$} & \multirow{2}{*}{$\begin{array}{c}\text { Over } \\
\text { all } \\
\%\end{array}$} \\
\hline & & & & $\mathbf{Y}$ & $\mathbf{N}$ & $\mathbf{Y}$ & $\mathbf{N}$ & $\mathbf{Y}$ & $\mathbf{N}$ & & \\
\hline \multirow{2}{*}{1} & \multirow{2}{*}{ MCL } & SSG IM + Allopurinol & 14 & 9 & 5 & 2 & 3 & 0 & 3 & 78.6 & \multirow{2}{*}{82.2} \\
\hline & & SSG IM + IL & 14 & 8 & 6 & 4 & 2 & 0 & 2 & 85.7 & \\
\hline \multirow{2}{*}{2} & \multirow{2}{*}{ DCL } & SSG IM + Allopurinol & 10 & 3 & 7 & 3 & 4 & 2 & 2 & 80 & \multirow{2}{*}{82.9} \\
\hline & & SSG + Cryotherapy + IL & 7 & 4 & 3 & 2 & 1 & 0 & 1 & 85.7 & \\
\hline \multirow{2}{*}{3} & \multirow{2}{*}{ LCL } & Cryotherapy & 26 & 17 & 9 & 6 & 3 & 1 & 2 & 92.3 & \multirow{2}{*}{94.2} \\
\hline & & IL + Cryotherapy & 26 & 12 & 14 & 8 & 6 & 5 & 1 & 96.1 & \\
\hline
\end{tabular}

$85.7 \%$. This result was consistent with a study in Silti (Southern Ethiopia) (85\%) and a report from WHO (82\%) [2,12]. This might be since both researches were conducted on old world cutaneous leishmaniasis cases with administration of parental SSG and local therapy (IL SSG). But the result was relatively low when compared to a study conducted in the United States and report from Medicines and Health care products Regulatory Agency $(90 \%)[13,14]$. This might be due to different etiological agents that cause leishmaniasis in New World and Old World. But to the contrary the result was too high relative to the research done in ALERT-Ethiopia though the causative agent and geographical location and treatment pattern is the same. Such a difference might be due to duration of treatment. The ALERT study was conducted for a month or a cycle but this study was done for three cycles for three months .On the other way round, those patients who took systemic SSG with allopurinol showed a cure rate of $78.6 \%$. This result was relatively higher with the study conducted in Saudi Arabia (71\%) [13]. This difference might be because of the etiologic agents and treatment phases or cycles $[3,1]$.

Patients with Diffused Cutaneous Leishmaniasis (DCL), who have took systemic sodium stibogluconate via intra muscular or/ and intravenous with allopurinol showed the curative rate was as high as $80 \%$. This result was relatively higher with the research conducted in Tigray-Ethiopia (75\%). This might be due to treatment cycle and application of different herbs. In this study patient took medication for three cycles but one cycle in Tigray where as nearly half of the study subjects in Tigray and 34\% in Boru Meda had herb application. But those who took systemic SSG and combined local therapy both cryotherapy and IL SSG had a clinical cure rate of $85.7 \%$. This result was relatively lower when compared to the study conducted in United Arab Emirate (100\%) (13). This might be due to different strains of causative agents, application technique (using cryo gun vs cotton tipped) and one third of the cases had history of herb application in our clinic.

A total of 52 patients who diagnosed as localized cutaneous leishmaniasis and took local therapy provide different clinical improvements in a combined and single treatment pattern. Twenty-six patients who had only cryotherapy showed clinical cure rate of $92.3 \%$. At the 3rd dose only two patients had very good improvement but few satellite popular lesions around the mother lesions. This was relatively higher from the study conducted in United Arab Emirates (68\%), Turkish study (68\%) and Tigray (60-70\%) [13,15,3]. Such a difference might be due to duration and numbers of applications i.e. 12 weeks of application vs 6 weeks and 6-8 times, application technique (using cotton tipped vs cryo gun) and size of lesions. But to the contrary our result is relatively low when compared to trials in Egypt (100\%) [16]. this might be due to application of cryotherapy for undetermined times, till all lesions are cleared. In addition, other groups containing 26 patients had a combined cryotherapy and IL SSG therapy with a cure rate of $96.1 \%$. This result was relatively higher when compared to report from Turkey $(81.8 \%)$ and Colombia $(68 \%)[17,18]$. This might be due to numbers of cryotherapy application (6-8 vs 2-4 times) and application of IL and cryotherapy (we applied cryotherapy 2-3 days of IL SSG vs application of both local at the same time).

\section{Conclusions}

Cutaneous leishmaniasis is a neglected tropical disease overshadowed by lack of effective anti-leishmaniasis agent in Ethiopia. Thus, high number of population is faced for various degree of socio-economical and psychosocial morbidity.

As our study showed a combined anti- leishmaniasis agents like SSG IM with IL, SSG IM with local therapies (cryotherapy + IL SSG), cryotherapy only or combined both local therapies (cryotherapy + IL SSG) results 85.7\% (MCL), 85.7\% (DCL)\&\%, 92.3\% (LCL)and 96.1\% (LCL) effectiveness for respective clinical category, respectively.

Thus, for any clinical type of cutaneous leishmaniasis administering combined forms (Pentavalent antimonial with local therapies i.e. cryotherapy or/and IL SSG) of anti-leishmaniasis agents had a better cure rate than single therapies.

\section{References}

1. Postigo JA. Leishmaniasis in the World Health Organization Eastern Mediterranean Region. Int J Antimicrobial Agents. 2010;36 Suppl 1:S62-S65. doi: 10.1016/j.ijantimicag.2010.06.023

2. Negera E, Gadisa E, Yamuah L, Engers H, Hussein J, Kuru T, et al. outbreak of cutaneous leishmaniasis in Siltiworeda, Ethiopia: risk factor assessment and causative agent identification. Transactions of the royal society of tropical medicine and hygiene. 2008;102(9):883-890. 
3. Morrone A1, Pitidis A, Pajno MC, Dassoni F, Latini O, Barnabas GA, et al, A Epidemiological and geographical aspects of leishmaniasis in Tigray, northern Ethiopia: a retrospective analysis of medical records, 2005-2008. Trans R Soc Trop Med Hyg. 2011;105(5):273280. doi: 10.1016/j.trstmh.2011.02.003

4. Reithinger R, Dujardin JC, Louzir H, Pirmez C, Alexander B, Brooker S. Cutaneous leishmaniasis. Lancet Infect Dis. 2007;7(9):581-596.

5. World Health Organization. Global plan to combat neglected tropical diseases 2008-2015. 2009.

6. Croft SL, Seifert K, Yardley V. Current scenario of drug development for leishmaniasis. Indian J Med Res. 2006;123(3):399-410.

7. Croft SL, Sundar S, Fairlamb AH. Drug resistance in leishmaniasis. Clin Microbiol Rev. 2006;19(1):111-126.

8. SimeenberRehman, Arfan-ul-Bari. Laboratory profile in patients of cutaneous leishmaniasis from various regions of Pakistan. J Coll Physicins Surg Pak. 2003;13(6):313-316.

9. Alvar J, Vélez ID, Bern C, Herrero M, Desjeux P, Cano J, et al. Leishmaniasis worldwide and global estimates of its incidence. PLoS One. 2012;7(5):e35671. doi: 10.1371/journal.pone.0035671

10. Leishmaniasis mapping team at AHRI in collaboration with WHOEthiopia. Proceedings of the international consultative meeting on cutaneous leismaniasis in Ethiopia; Addis Ababa, July 4-5, 2011.

11. Negera E, Gadisa E, Hussein J, Engers H, Kuru T, Gedamu L, et al Treatment response of cutaneous leishmaniasis due to Leishmania aethiopica to cryotherapy and generic sodium stibogluconate from patients in Silti, Ethiopia. Trans R Soc Trop Med Hyg. 2012;106(8):496-503. doi: 10.1016/j.trstmh.2012.02.006
12.WHO. Report of a meeting of the WHO Expert Committee on the Control of Leishmaniases, Geneva, Switzerland, 22-26 March 2010. WHO technical report series. 2010;949.

13.Wortmann G, Miller RS, Oster C, Jackson J, Aronson N. A randomized, double-blind study of the efficacy of a 10- or 20-day course of sodium stibogluconate for treatment of cutaneous leishmaniasis in United States military personnel. Clin Infect Dis. 2002;35(3):261-267.

14. Medicines and Health care products Regulatory Agency. Drug AlertPentostam Injection. MDR 47-03/66 dated 11 April 2006.

15.Layegh P, Pezeshkpoor F, Soruri AH, Naviafar P, Moghiman T. Efficacy of cryotherapy versus intralesionalmeglumineantimoniate (glucantime) for treatment of cutaneous leishmaniasis in children. Am J Trop Med Hyg. 2009;80(2):172-175.

16.Gurei MS, Tatli N, Ozbilge H, Erel O, Seyrek A, Kocyigit A, et al. Efficacy of cryotherapy and intralesionalpentostam in treatment of cutaneous leishmaniasis. J Egypt Soc Parasitol. 2000;30(1):169-176.

17. Brito NC, Rabello A, Cota GF, Efficacy of pentavalent antimoniateintralesional infiltration therapy for cutaneous leishmaniasis: A systematic review. PLoS One. 2017;12(9):e0184777. doi: 10.1371/journal.pone.0184777

18. Wortmann G, Miller RS, Oster C, Jackson J, Aronson N. A randomized, double-blind study of the efficacy of a 10- or 20-day course of sodium stibogluconate for treatment of cutaneous leishmaniasis in United States military personnel. Clin Infect Dis. 2002;35(3):261-267. 